\title{
Steroid versus Non-Steroid Regimen in Treating Duchenne Muscular Dystrophy: An Observational Study from South India
}

\author{
N. C. SATTENAPALLI*, A. R. ARETI, S. N. K. R. GUDHANTI, U. KULANDIVELU AND R. R. ALAVALA \\ KL College of Pharmacy, Koneru Lakshmaiah Education Foundation, Vaddeswaram, Guntur, Andhra Pradesh 522502, India
}

Sattenapalli et al.: Steroid versus Non-Steroid Regimen in Duchenne Muscular Dystrophy

\begin{abstract}
There are no large studies from Southern India describing the treatment regimen of the Duchenne muscular dystrophy which is the most common muscular dystrophy. The current observational study aimed to compare steroid versus non-steroid treatment outcomes in Duchenne muscular dystrophy diagnosed population of South India. Immunohistochemically/genetically confirmed Duchenne muscular dystrophy patients diagnosed between February 2019 and March 2021 were ambispectively included. The main outcomes assessed in the study were loss of ambulation with respect to age, disease progression of motor milestones, medical research council grade, change in body weight and reported adverse events. We correlated these outcomes comparatively for both intervention groups (steroid and non-steroid). A total of 274 Duchenne muscular dystrophy patients were included in the study. The mean minimal age of onset of disease was $2.0 \pm 1.2$ y. $32.85 \%$ patients were treated with steroids and $67.15 \%$ patients were on non-steroidal treatment. Comparatively, both regimen using patients demonstrated loss of ambulation at 13 y of age. Gait, stairs, Gower, chair scores reported loss of motor functions at 13 y and 25 y for steroid and non-steroid using patients respectively. Medical research council grade improved in steroid using patients to a greater extent compared to non-steroid using patients. Change in body weight and adverse events were comparatively low in non-steroid using patients to that of steroid using patients. The pattern of primary outcome measures in this large cohort is comparable despite variability in medical care. The contemporary observational study adds to the real-world evidence in guiding clinicians towards better approach in treating Duchenne muscular dystrophy patient populationa.
\end{abstract}

Key words: Steroids vs. non-steroids, Duchenne muscular dystrophy, Gait, stairs, Gower, chair scores, loss of ambulation, motor functions

Duchenne Muscular Dystrophy (DMD) is an X-linked recessive inherited neuromuscular dysfunction resulting from mutations in the dystrophin gene which codes for dystrophin, a cytoplasmic protein found at muscle membranes. Dystrophin plays a key role in regulating the actin cytoskeleton ${ }^{[1]}$. In early childhood, depending on the type of dystrophin gene mutation, there may be a marked decline or absence of dystrophin in muscle, resulting in $\mathrm{DMD}^{[2]}$. This condition is associated with progressive muscle weakness and extreme wasting caused by lack of dystrophin protein, which results in loss of ambulation, total wheelchair dependency by the age of $13 \mathrm{y}^{[3-5]}$. The endogenous inflammatory response, such as Nuclear Factor kappa-B (NF- $\kappa \mathrm{B})$ activation, which is detectable in dystrophin-deficient muscle, exacerbates skeletal and cardiac muscle degeneration ${ }^{[6]}$ leading to premature death.

*Address for correspondence

E-mail: nigamac111@gmail.com

January-February 2022
Althoughrare,DMD is the most prevalent neuromuscular disorders and muscular dystrophy in early childhood, affecting one out of every 3500 newborn males ${ }^{[7,8]}$. If left untreated, combination of muscle fatigue and tendon contractures in the tendon Achilles and iliotibial bands contributes to loss of independent walking by the age of $9.5 \mathrm{y}$ (range 7 to $13 \mathrm{y})^{[9]}$. With improvement in medical management especially in respiratory and cardiac treatment, life-expectance of DMD patients has increased up to $30-40 \mathrm{y}$.

\footnotetext{
This is an open access article distributed under the terms of the Creative Commons Attribution-NonCommercial-ShareAlike 3.0 License, which allows others to remix, tweak, and build upon the work non-commercially, as long as the author is credited and the new creations are licensed under the identical terms
}

Accepted 25 January 2022

Revised 05 July 2021

Received 03 February 2021

Indian J Pharm Sci 2022;84(1):80-86 
Due to the distortion of the reading frame caused by variants in the dystrophin gene, pharmacological therapies are only partially efficient culminating in a significant unmet clinical need. Although there is no effective treatment for DMD at present, glucocorticoids (prednisone, deflazacort) are the standard of care in DMD due to their anti-inflammatory effects, which tend to mitigate the onset of symptoms ${ }^{[10,11]}$. Unfortunately, despite the therapeutic benefits, chronic glucocorticoid therapy causes a wide range of side effects, including bone fragility, stunted growth, weight gain, behavioral problems, cataracts and adrenal suppression, each of which diminish patient quality of life ${ }^{[12,13]}$. These negative side effects trigger extension of treatment, non-adherence and variations in clinical practice ${ }^{[12,14]}$.

There is currently no consensus on which treatment offers the most effective treatment with the fewest side effects. It has been reported that the regimens used in practice still vary in India ${ }^{[15]}$, all of which might have been recommended by experts in the past. Studies reporting the clinical practice for DMD and long-term follow-up data from India is lacking in literature. The present work was an attempt to study the comparative treatment regimen for the disease and its survival pattern in a large cohort of DMD population from India. The observational data dawns the current intervention strategy to pediatric therapists across different child diagnostic populations of DMD in Southern India. In context to this lacuna, we conducted an observational study on DMD diagnosed patients and compared their treatment regimen in collaboration with the Amaravathi Muscular Dystrophy Association (AMDA) to elucidate the epidemiology, clinical practice and compare steroids and non-steroids regimen in these patient population of South India.

\section{MATERIALS AND METHODS}

\section{Study design and patient selection:}

This was an observational study with retrospective patient recruitment from the AMDA [Registration. No. 33 of 2019] which maintains the data set of muscular dystrophy patients in India. The AMDA was established in 2019 to facilitate scientific communication and collaboration in the neuromuscular disease field in South India and is likely to have the largest clientele to capture DMD cases in their locale. Patients were identified retrospectively by retrieving case records of those diagnosed with DMD either genetically (multiplex polymerase chain reaction/multiplex ligationdependent probe amplification or both) or by muscle immunohistochemistry (biceps or quadriceps biopsy) between February 2019 and March 2021. Written informed consent for participation in the study was provided by all patients or their legal representatives. A total of 274 patients who had furnished details of postal address were sent a simple questionnaire in English and local language along with return paid post. The clinical details were collected till March 2021 and entered in a pre-designed proforma.

\section{Data collection:}

Data was collected and analysed in Microsoft Excel 2016 (Redmond, Washington, United States of America (USA)) and GraphPad Prism version 9.1.0. (GraphPad Software, Inc., San Diego, California). The deidentified data were curated for the following variables of interest: Demographics, vitals, treatment regimen in use, glucocorticoid steroid in case of steroidal treatment and grades of motor functions. Patients included in the present study were required to have a recorded date of steroid initiation. The outcomes of interest were loss of ambulation, milestones of disease progression of motor functions (in terms of gait, climbing stairs, Gower's maneuver and arising from a chair (GSGC)), Medical Research Council (MRC) grade, change in body weight and adverse events.

\section{Questionnaire:}

The survey consisted of 3 questions of patient's background and 11 questions of clinical practice on DMD. Questions regarding clinical practice for DMD probed are the number of patients with DMD grouped by age (0-10 y, 11-20 y, >21 y); awareness of DMD care recommendations published in 2010 $0^{[16]}$; steroid/ non-steroid therapy experience; patient's loss of ambulation status; GSGC score; MRC grade; body weight; experience of adverse events during therapy. We extracted responses from the patients who had either steroid or non-steroid therapy experience and analysed their habits and practices regarding therapy followed (steroid or non-steroid therapy).

\section{RESULTS AND DISCUSSION}

In February 2019, we contacted the AMDA executive board members of South India to acquire their agreement to conduct observational study to compare steroidal and non-steroidal regimen used in South India. All patients belonged to South India. In terms of the steroid regimen deflazacort was the most common (dose range: $5-20 \mathrm{mg} / \mathrm{kg} / \mathrm{d}$ ) and other steroid being, prednisolone (dose range: $3-30 \mathrm{mg} / \mathrm{kg} / \mathrm{d}$ ). 
Table 1 presents the reported number of patients with DMD and those treated with steroids and nonsteroids, by age group. Among the 274 participants, $32.85 \%$ patients were treated with steroids and 67.15 $\%$ patients were on non-steroidal treatment. Regarding the distribution of patients according to age groups; 0-5 y, 6-10 y, 11-15 y, 16-20 y, 21-25 y, 26-30 y and $31-35$ y were $1.11 \%, 21.11 \%, 55.55 \%, 14.44 \%$, $6.67 \%, 0 \%, 1.11 \%$ for steroidal patients and $3.26 \%$, $14.13 \%, 46.74 \%, 25 \%, 8.70 \%, 2.17 \%$ and $0 \%$ for non-steroidal patients respectively (fig. 1).

Kaplan-Meier plot of loss of ambulation relative to age (y), grouped by participants treated with steroids $(n=90$, red line) $v s$. participants with non-steroidal treatment

\section{TABLE 1: STEROIDS vS. NON-STEROIDS} PERCENTAGE OF PATIENTS BY AGE

\begin{tabular}{lcc}
\hline Age $(\mathbf{y})$ & \% of steroidal patients & $\begin{array}{c}\text { \% of non-steroidal } \\
\text { patients }\end{array}$ \\
\hline $0-5$ & 1.11 & 3.26 \\
$6-10$ & 21.11 & 14.13 \\
$11-15$ & 55.55 & 46.74 \\
$16-20$ & 14.44 & 25 \\
$21-25$ & 6.67 & 8.70 \\
$26-30$ & 0 & 2.17 \\
$31-35$ & 1.11 & 0 \\
\hline
\end{tabular}

Steroids vs. non-steroids percentage of patients by age

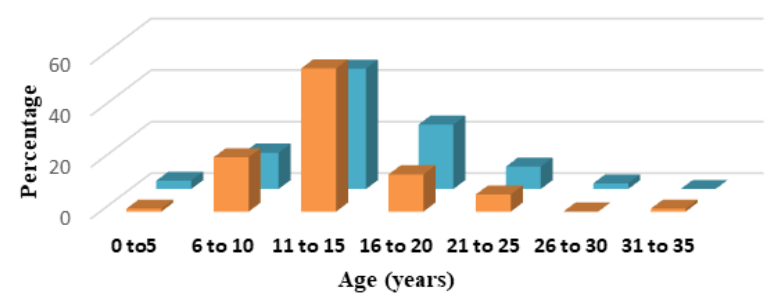

Fig. 1: Steroids vs. non-steroids percentage (\%) of patients by age, $\left(^{-}\right) \%$ of steroid patients; $\left(^{\square}\right) \%$ of non-steroid patients
( $\mathrm{n}=184$, blue line) (fig. 2). Comparatively, both regimen using patients demonstrated loss of ambulation at $13 \mathrm{y}$ irrespective of the interventions used. However, it is noteworthy steady ambulatory status with sudden drop in ambulation for steroids using patients. In case of non-steroid using patients, onset of ambulatory status distortion started at $2 \mathrm{y}$ with steady rate in loss of ambulation.

Milestones of disease progression in terms of motor functions were described below. Disease progression was expressed in terms of scores gained by patients for four motor functions viz. Gait (G), climbing Stairs (S), Gower's maneuver (G) and arising from a Chair (C). GSGC scores define the disease progression of patients. Kaplan-Meier plot (fig. 3 and fig. 4) of disease progression of motor function relative to age $(\mathrm{y})$, grouped by $\mathrm{G}$ (blue line), S (red line), G (green line) and $\mathrm{C}$ (purple line) scores for both participants using steroids $(\mathrm{n}=90)$ vs. participants with non-steroidal treatment $(n=184)$. The plot depicts disease progression which clearly indicates the steroid using patients had better quality of life due to late onset of disease progression unlike non-steroidal patients. Disease progression started with disruption in Gait scores, followed by $\mathrm{C}$, $\mathrm{S}$ and G-scores subsequently in both steroid using and non-steroidal patients. However, as age progressed patients lost the motor functions at the age of $13 \mathrm{y}$ on average in steroid using patients. Whereas, non-steroid using patients lost motor functions in a differential manner starting from Gower's maneuver at age $10 \mathrm{y}$, while S-scores in 13 y followed with loss in G-score at $16 \mathrm{y}$ age and subsequently lost $\mathrm{C}$-scores at $25 \mathrm{y}$ age. It is noteworthy, that disease progressed at a lower rate in patients with non-steroid treatment on contrary to the steroid using patients.

Comparative study for both the regimen used by patients, were plotted and shown in fig. 5. Steroid

\section{Loss of Ambulation}



Fig. 2: Steroid vs. non-steroids loss of ambulation, ( $\bullet$ ) Nonsteroid patients; $(-$ - ) Steroid patients 


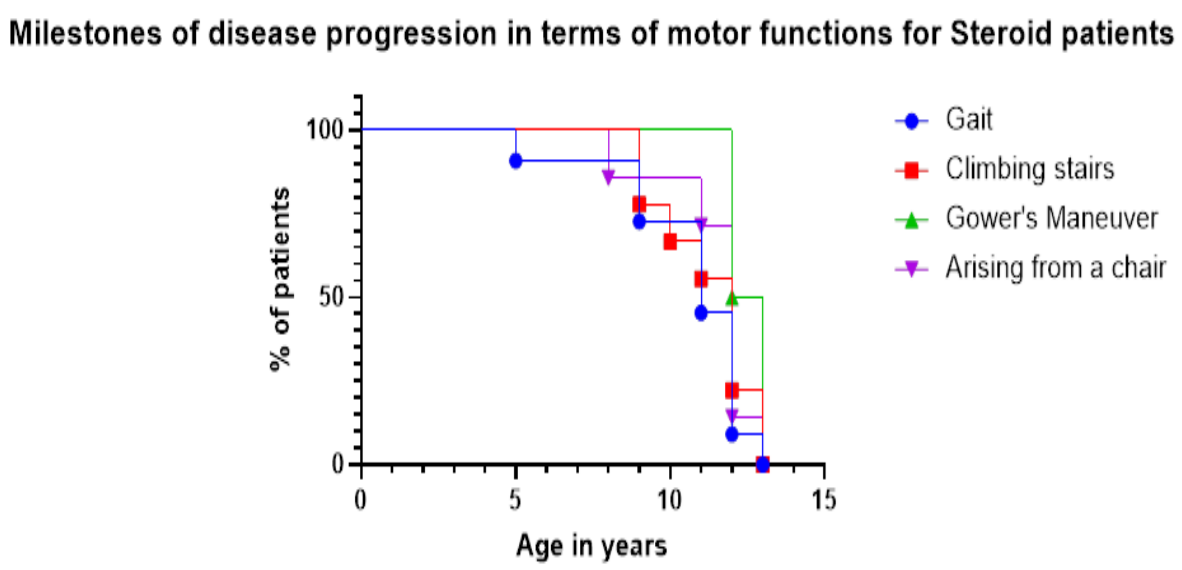

Fig. 3: Milestones of disease progression for steroid patients, $\left(-\bullet^{-}\right)$Gait; $\left(^{-}-\right)$Climbing stairs; $\left(-^{-}\right)$Gower's maneuver; $(\neg)$ Arising from a chair

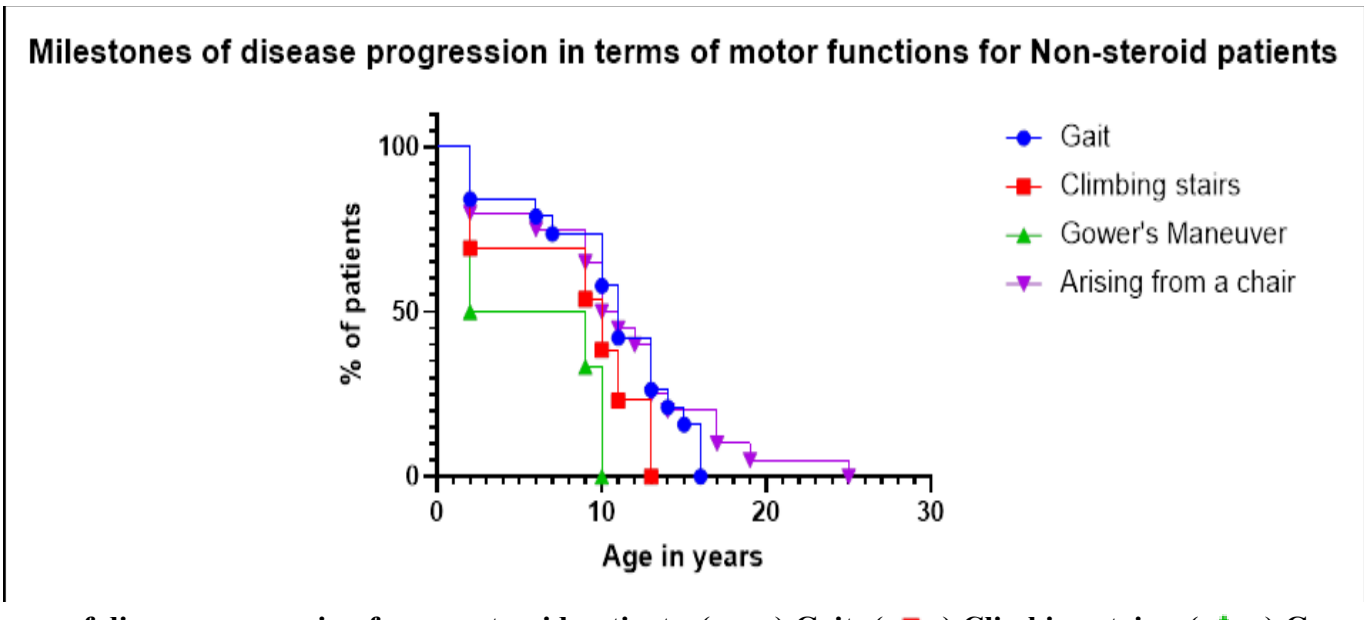

Fig. 4: Milestones of disease progression for non-steroid patients, $\left(--^{-}\right)$Gait; $\left(^{--}\right)$Climbing stairs; $\left({ }^{-}\right)$) Gower's maneuver; $(\rightarrow)$ Arising from a chair

\section{MRC grade for non-steroid and steroid treated patients}

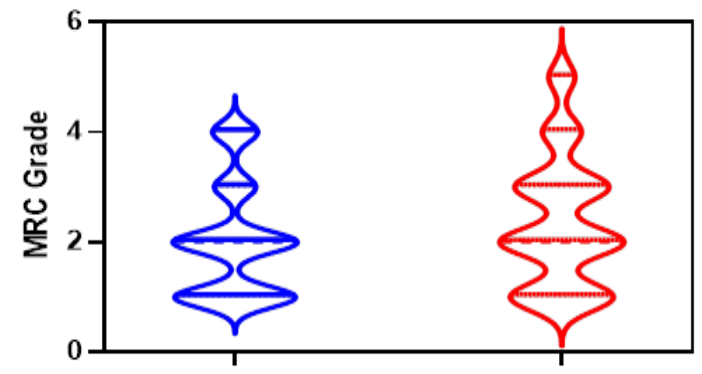

Age in years

Fig. 5: Steroid vs. non-steroids MRC grade, (ש) Non-steroid patients; (匚 ) Steroid patients

using patients reported better MRC grade compared to non-steroidal patients wherein, worsening of MRC grade started at an early age. Based on MRC grade, steroid using patients showed stabilized strength and improvement in movement unlike non-steroid using patients score.
Non-steroid treatment showed gradual body weight increase which could be due to general growth process of patients. While in steroid using patients, there is a drastic increase in body weight irrespective of growth process (fig. 6). Steroid using patients showed a maximum of $17 \%$ increase in body weight whereas, 
non-steroid using patients reported less than $10 \%$ change in body weight.

Both steroid using and non-steroid using patients reported most of the related adverse events listed in Table 2. Both the treatment groups reported gastric symptoms at a major rate followed by appetite increase in 11-20 y aged patients. Steroid using patients reported all adverse events but, non-steroid using patients did not report any behavioural changes, glycosuria, blood pressure and skin discoloration. Percentages of adverse events reported by patients in both intervention groups were tabulated briefly in Table 2.

Currently, increasing number of research papers regarding patient's epidemiology and clinical practice in DMD cases have been published from countries using a research infrastructure. Nonetheless, relevant research evidence is still lacking in Asian countries, especially in India; also, research infrastructures in rare diseases have been underdeveloped. Accordingly, this study was carried out to investigate the current clinical practice with respect to comparative effect of treatment regimen (steroid vs. non-steroids) for DMD in South India, in collaboration with the AMDA.

An observational study was performed to compare the current clinical practice among DMD patients using steroids with non-steroid using patients. Disease was diagnosed reportedly on an average of 2 y age; wherein major number of patients were at the range of 11-15 y age. Deterioration of ambulatory status had an early onset in non-steroid using patients, while patients with steroidal treatment had delayed loss of ambulation which indicates the usage of steroids

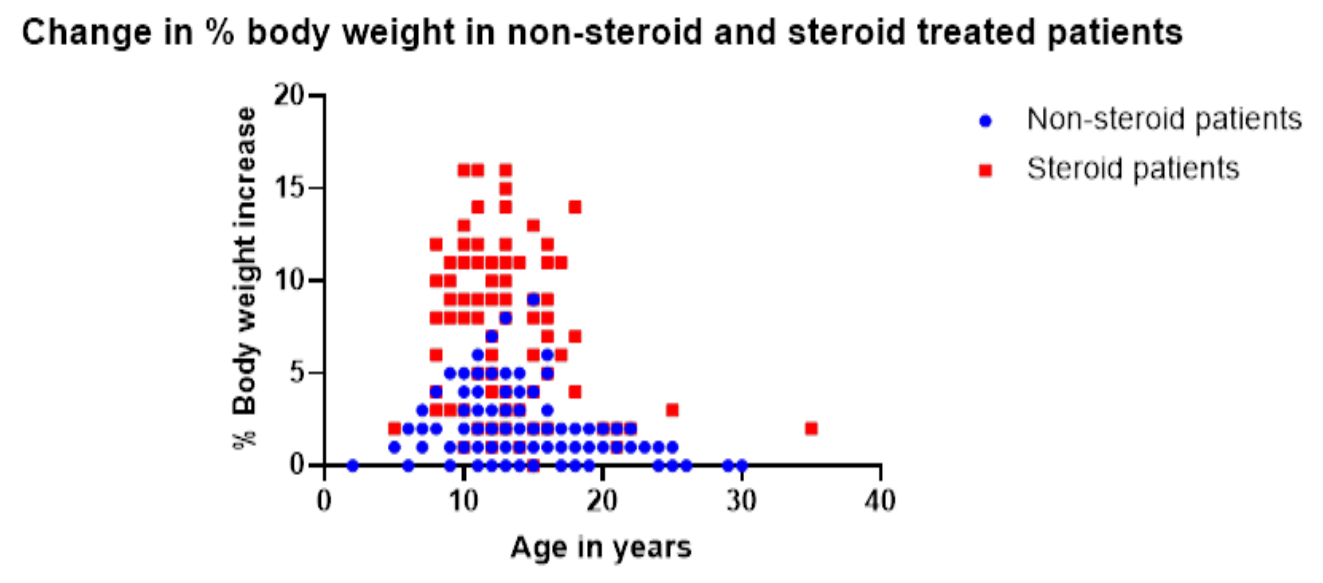

Fig. 6: Steroid vs. non-steroids change in \% body weight, $(\bullet)$ Non-steroid patients; $(=)$ Steroid patients

TABLE 2: STEROIDS vs. NON-STEROIDS PERCENTAGE OF ADVERSE EVENTS REPORTED BY AGE

\begin{tabular}{lcccc}
\hline \multirow{2}{*}{ Adverse events } & Regimen & \multicolumn{3}{c}{ Age (y) } \\
\cline { 3 - 5 } Cushingoid appearance & NST & $\mathbf{0 - 1 0}$ & $\mathbf{1 1 - 2 0}$ & $\mathbf{2 2 1}$ \\
\hline \multirow{2}{*}{ Gastric symptoms } & ST & 12.5 & 9.85 & 5 \\
& NST & 35 & 28.57 & 42.86 \\
Appetite increase & ST & 53.16 & 34.85 & 30 \\
& NST & 65 & 76.19 & 57.14 \\
Behavioural changes & ST & 6.25 & 6.06 & 10 \\
& NST & 65 & 68.25 & 57.14 \\
Glycosuria & ST & 0 & 2.27 & 0 \\
Blood pressure & NST & 10 & 3.17 & 28.57 \\
Skin discoloration & ST & 0 & 0 & 0 \\
& NST & 5 & 9.52 & 14.29 \\
\hline
\end{tabular}

Note: NST-Non-steroid treatment; ST-Steroid treatment 
enhances the patient's ambulatory status. Milestones of disease progression were calculated in terms of GSGC scores for both the intervention groups. Steroid using patients reported delayed disease progression, whereas, in non-steroid using patients reported deterioration of motor functions at an early stage but complete loss of motor functions progressed till $25 \mathrm{y}$. In contrary, steroid using patients lost motor functions at less than $15 \mathrm{y}$ of age. This indicates that usage of steroids surely delays the onset of progression of disease but non-steroidal treatment makes the patient adaptable to withstand and delay the loss of motor functions. These GSGC scores can be correlated with the loss of ambulation. Delayed onset of disease progression can be correlated to improved ambulatory status at the beginning in steroid using patients and complete deterioration of motor functions leading to loss of ambulation. The same is the scenario in non-steroid using patients who reported loss of ambulation at early stage. Body weight was drastically increased in steroid using patients, unlike non-steroid using patients. This could be the side-effects of steroids which is susceptible in enhancing the body weight of patients during its usage. Steroid using patients reported improved MRC grade compared to non-steroid using patients who could be due to the effect of steroid interventional influence on patient improving the muscle strength and decreasing inflammation. Major adverse events were reported by patients using steroidal treatment compared to the nonsteroid using patients. These events were at differential rate, which could be due to the interventional influence.

The age, delay in disease progression milestones, motor functions, GSGC scores and MRC grade all showed significant impact on the ambulatory status. Mirski et $a l .{ }^{[17]}$ found that delay in the onset of walking in boys with DMD is strongly associated with a cognitive delay; however, the impact of the latter on loss of ambulation was not discussed. Most of the parents/ caregivers preferred physiotherapy or alternative medicine only because of their personal preferences, local convictions, cultural preconceptions and an unwarranted apprehension towards the adverse effects of treatment regimen, and the ineffectiveness of most of medications in treating them.

Our study consisted DMD diagnosed children from lower socioeconomic status and usually residing in rural areas. The main reason for the delay in diagnosis might be due to the less access to medicines and neurologists. In addition, the low literacy rate among parents and caretakers might have led to the delay in seeking medical advice. On instances, we have observed poor clinical suspicion by the primary care physicians resulting in delayed referral/diagnosis. The delay in motor function milestones were usually considered as constitutional delay or other unknown causes attributed to them without an affected child being involved in the family.

The contemporary observational study lights on the missing epidemiological data, current practice and comparative study on steroids with non-steroid treatment in DMD patients of South India which could guide the physicians in prescribing treatment regimen and improving quality of life of DMD patients.

To our knowledge, this is the first study from South India comparing the treatment regimen and current practice of DMD in a large cohort of genetically and/or immunohistochemically confirmed cases. The patterns of major DMD milestones, including the age, age at loss of ambulation and adverse events in our study are reported despite variability in the medical care. Oral steroids delay the loss of ambulation and probably add quality years to the life of the patients suffering from DMD while non-steroidal treatment reduces adverse events, change in body weight along with delayed disease progression however with compromised quality of life. This study also highlights the current scenario of obstacles involved in establishing the diagnosis of DMD and its management. We suggest that it is important to expand the registries of patients with DMD in India and to accumulate real-world longitudinal patient data. These strategies will aid the study of the epidemiology and current clinical practice of DMD and could improve treatment and care for patients with DMD worldwide.

\section{Acknowledgments:}

The authors are thankful to College of Pharmacy, Koneru Lakshmaiah Education Foundation, Vaddeswaram, Andhra Pradesh, India, for providing facilities to carry out the research.

\section{Conflict of interests:}

The authors have no conflicts of interest relevant to this article to disclose.

\section{REFERENCES}

1. Hoffman EP, Brown Jr RH, Kunkel LM. Dystrophin: The protein product of the Duchenne muscular dystrophy locus. Cell 1987;51(6):919-28.

2. Koenig M, Beggs AH, Moyer M, Scherpf S, Heindrich K, Bettecken T, et al. The molecular basis for Duchenne versus Becker muscular dystrophy: Correlation of severity with type 
of deletion. Am J Hum Genet 1989;45(4):498-506.

3. Emery AE. The muscular dystrophies. Lancet 2002;359:68795.

4. Fairclough RJ, Wood MJ, Davies KE. Therapy for Duchenne muscular dystrophy: Renewed optimism from genetic approaches. Nat Rev Genet 2013;14(6):373-8.

5. Chen YW, Nagaraju K, Bakay M, McIntyre O, Rawat R, Shi $\mathrm{R}$, et al. Early onset of inflammation and later involvement of TGF $\beta$ in Duchenne muscular dystrophy. Neurology 2005;65(6):826-34.

6. Messina S, Vita GL, Aguennouz M, Sframeli M, Romeo S, Rodolico C, et al. Activation of NF-kB pathway in Duchenne muscular dystrophy: Relation to age. Acta Myol 2011;30(1):1623.

7. Mendell JR, Shilling C, Leslie ND, Flanigan KM, al-Dahhak $\mathrm{R}$, Gastier-Foster J, et al. Evidence-based path to newborn screening for Duchenne muscular dystrophy. Ann Neurol 2012;71(3):304-13.

8. Moat SJ, Bradley DM, Salmon R, Clarke A, Hartley L. Newborn bloodspot screening for Duchenne muscular dystrophy: 21 y experience in Wales (UK). Eur J Hum Genet 2013;21(10):1049-53.

9. Bushby K, Finkel R, Birnkrant DJ, Case LE, Clemens PR, Cripe L, et al. Diagnosis and management of Duchenne muscular dystrophy, part 1: Diagnosis and pharmacological and psychosocial management. Lancet Neurol 2010;9(1):7793.

10. Birnkrant DJ, Bushby K, Bann CM, Apkon SD, Blackwell A,
Brumbaugh D, et al. Diagnosis and management of Duchenne muscular dystrophy, part 1: Diagnosis and neuromuscular, rehabilitation, endocrine, and gastrointestinal and nutritional management. Lancet Neurol 2018;17(3):251-67.

11. McDonald CM, Henricson EK, Abresch RT, Duong T, Joyce NC, Hu F, et al. Long-term effects of glucocorticoids on function, quality of life, and survival in patients with Duchenne muscular dystrophy: A prospective cohort study. Lancet 2018;391(10119):451-61.

12. Bello L, Gordish-Dressman H, Morgenroth LP, Henricson EK, Duong T, Hoffman EP, et al. Prednisone/prednisolone and deflazacort regimens in the CINRG Duchenne natural history study. Neurology 2015;85(12):1048-55.

13. Ma J, McMillan HJ, Karagüzel G, Goodin C, Wasson J, Matzinger MA, et al. The time to and determinants of first fractures in boys with Duchenne muscular dystrophy. Osteoporos Int 2017;28(2):597-608.

14. Griggs RC, Herr BE, Reha A, Elfring G, Atkinson L, Cwik V, et al. Corticosteroids in Duchenne muscular dystrophy: Major variations in practice. Muscle Nerve 2013;48(1):27-31.

15. Arora H. Duchenne muscular dystrophy: Still an incurable disease. Neurol India 2019;67(3):717.

16. Medical Management-Duchenne Muscular Dystrophy (DMD)Diseases. Muscular Dystrophy Association; 2017.

17. Mirski KT, Crawford TO. Motor and cognitive delay in Duchenne muscular dystrophy: Implication for early diagnosis. J Pediatr 2014;165(5):1008-10. 